\title{
RESISTENCIA AL CAMBIO $Y$ SU INFLUENCIA EN LA IDENTIFICACIÓN INSTITUCIONAL DEL PERSONAL DE LA CAJA MUNICIPAL DE AHORRO Y CRÉDITO CUSCO S.A. - AGENCIA TACNA, EN EL AÑO 2013
}

\author{
Resistance to change and its influence on institutional staff \\ identification CMAC Cusco SA - Tacna agency in the year 2013
}

\section{María Isabel Apaza Chambilla²}

\section{RESUMEN}

Objetivo: Esta investigación tiene como objetivo principal el determinar la influencia del nivel de resistencia al cambio sobre la identificación institucional del personal de la Caja Municipal de Ahorro y crédito (CMAC) Cusco S.A. - Agencia Tacna para mejorar el servicio al cliente.

Método: El tipo de investigación es aplicada, cuyas características son descriptivas y correlacionales. EI diseño de la investigación es no experimental. La recolección de la información fue de corte transversal. Se optó por desarrollar un censo, se encuestaron a las 40 personas, tanto del Área de Operaciones y Área de Créditos.

Resultados: Se encontró que los trabajadores perciben que existe un nivel bajo de resistencia ante posibles entornos de cambio en la organización; siendo los "Factores asociados al saber/conocer" los que afectan menos y los "Factores organizacionales asociados al proyecto de cambio" los que afectan más. También se encontró que el personal considera que se encuentra muy identificado con su entidad; siendo la "Realización" la dimensión más destacada, y el "Compromiso" el aspecto a fortalecer.

Conclusión: Existe una relación significativa entre las dimensiones del nivel de resistencia al cambio y las dimensiones de la identificación institucional del personal.

\section{PALABRAS CLAVES}

Competitividad, cambio, función administrativa, motivación, identificación institucional.

\section{ABSTRACT}

Objective: This Tiene Investigación Objetivo Como director el determinar S. La influencia del Nivel de Resistencia Al Cambio Sobre la identification institucional del personal de la Caja Municipal de Ahorro y Crédito (CMAC) Cusco SA - Agencia Tacna párrafo Mejorar el servicio al cliente.

Method: The research is applied, whose characteristics are descriptive and correlational. The research design is not experimental. The collection of information was crosssectional. It was decided to develop a census, they surveyed 40 people, both Area and Credit Division Operations.

\footnotetext{
${ }^{2}$ Magister en Administración y Dirección de Empresas
} 
Results: It was found that workers perceive that there is a low level of resistance to environments possible change in the organization; being the "Factors associated with know / meet" those affecting fewer and "Factors related to organizational change project" the most affecting. We also found that the staff believes that is closely identified with their entity; It is the "Placing" the most prominent dimension, and "commitment" to strengthen the look.
Conclusion: There is a significant relationship between the dimensions of the level of resistance to change and the dimensions of institutional personnel identification.

\section{KEYWORDS}

Competitiveness, however, administrative function, motivation, institutional identification.

\section{INTRODUCCIÓN}

Uno de los problemas principales que atraviesan las organizaciones, guardan relación con desarrollar formas de motivar al personal para que pongan más empeño en sus labores y aumentar la satisfacción e interés en el trabajo.

La función administrativa de la dirección se entiende como el proceso de influir en las personas para que contribuyan a los objetivos de la organización; para esto, es necesario que se den condiciones internas y externas, las condiciones internas se refieren a acontecimientos que ocurren dentro del trabajador, en especial el recuerdo y la activación de los conocimientos previos, en cambio las condiciones externas se refieren a sucesos del ambiente; pero tanto, el uno como el otro, incitarán a una respuesta natural del individuo, tomando una posición definida frente a cualquier hecho, ello se relaciona con la resistencia al cambio.

En la actualidad, uno de los problemas más urgentes que encaran las empresas es encontrar la manera de motivar al personal para que pongan más empeño en sus labores y aumentar la satisfacción e interés en el trabajo; puesto que las personas tienden a no aceptar un trabajo aburrido y cansado, por muy buen sueldo que les ofrezcan; ante ello, las empresas están obligadas a proporcionar trabajos más significativos, la cual es una manera de lograr un buen rendimiento y que el personal se sienta contento e interesado con el trabajo que desempeña.

En base a la experiencia laboral en la Caja Municipal de Ahorro y Crédito del Cusco S.A. - Agencia Tacna, se precisa que existen niveles preocupantes de desmotivación o disconformidad y de resistencia a los cambios del entorno; los cuales se resumen en dos aspectos:

- El personal del Área de Operaciones (Auxiliares de Operaciones) ve con recelo que los Analistas de Créditos y hasta Ejecutivos de Negocios, tienen mejores remuneraciones y mejor trato; además la existencia de algunas facilidades para realizar mejor la línea de carrera.

- En el Área de Negocios o Créditos existe desmotivación, puesto que la responsabilidad del trabajo implica la existencia de un estrés constante, 
alta presión y solo resultados, que consideran no va de la mano con el actual nivel remunerativo.

Complementando lo anterior, existe una problemática relacionada con la identificación institucional, ello se ve reflejado en la existencia de una tasa alta de rotación del personal; es decir, la "fuga de personal" es constante, bien por renuncia o por rescindirles los contratos; de forma más específica se precisa:

- El personal con frecuencia está a la expectativa de ofertas de trabajo, ya sea a través de medios impresos o electrónicos, cumplen estrictamente el horario de trabajo y lo exceden cuando el jefe se los propone.

- Aunque conocen y manejan la calidad tienden a no agregar valor al proceso, salvo que se les exija, pues su tarea consiste en dar respuesta a los requerimientos tal y como se le señalan.

- Son trabajadores promedio, destacan por la exactitud de su trabajo y la constancia, pero pocas veces por el impulso a los cambios y a las mejoras.

El cambio es un "Proceso a través del cual una organización llega a ser de modo diferente de lo que era en un momento dado anterior" (Soto, 2001). Todas las organizaciones cambian pero el reto que se plantean los directivos y, en general, todas las personas de la organización es que el cambio organizacional se produzca en la dirección que interesa a los objetivos de la organización. Es por ello, que se habla de gestión del cambio, agentes de cambio, intervención para el cambio, resistencia al cambio, etc.

El ambiente en general que envuelve a las organizaciones está en continuo movimiento y es dinámico, exige una elevada capacidad de adaptación de supervivencia. Deben enfrentarse a un entorno inestable, de cambio constante. Así, pues, para sobrevivir y competir hay que adaptarse al cambio rápida y eficazmente. El cambio que se realice, afectará en algún grado las relaciones de poder, estabilidad de roles y satisfacción individual al interior de la organización.

"Un proceso bien conducido de cambio implica lograr una transformación personal, que hace que el hombre este más alerta, más flexible y por eso muchas veces tiene que iniciar un análisis de revisión interior y de autoconocimiento. En este cambio, como proceso de aprendizaje permanente, debe involucrarse la alta gerencia (Lorenzo, 2000).

Para que un proceso de cambio pueda implementarse con éxito y sostenerse en el tiempo, es fundamental tener en cuenta el factor humano. Las personas deben confiar, estar motivadas y capacitadas, ya que el cambio es un proceso muy duro, tanto a nivel personal como organizacional. La persona que lidera el cambio debe lograr que las personas puedan hacer mejor el trabajo, con menor esfuerzo y mayor satisfacción. La confianza es un requisito esencial para lograr un ambiente de trabajo agradable y de franca cooperación.

Un aspecto importante a considerar es la tendencia natural de las personas de resistirse al cambio. Hay que crear y desarrollar una actitud y mentalidad abierta a los cambios, una cultura, que permita acoger las buenas iniciativas, así como desechar las malas. Los cambios organizacionales no deben dejarse al azar, ni a la inercia de la costumbre, menos a la improvisación, deben planificarse adecuadamente. Kurt Lewin, precisa tres causas comunes (Luperdi, 2007): 
a) Interés propio: Definidas como las razones personales que afectan o alimentan el deseo de cambio. Aquí se ubica la motivación, la costumbre a desarrollar un proceso definido de trabajo y la capacitación.

b) Cultura organizacional: Entendido como la fuerza fundamental que guía la conducta de los trabajadores: A veces, se sienten amenazados cuando se trata de efectuar cambios radicales en la manera de hacer las cosas en determinadas actividades.

c) Percepción de las metas y estrategias de la organización: Los miembros de un equipo no entienden que se necesita una meta nueva (un cambio), porque no cuentan con la misma información que manejan sus directivos.

El proceso de cambio planeado en las organizaciones induce el Desarrollo Organizacional, como un enfoque moderno de la organización para enfatizar en el cambio de actitud y de comportamiento de los sujetos que la conforman, en la cual, todos y cada uno de ellos debe estar dispuesto a participar de manera activa y consciente en la meta propuesta. El Desarrollo Organizacional es un proceso planificado, que promueve cambios de manera sistemática y organizada, proponiendo vías a seguir, en las fases de implementación, con la finalidad de minimizar la resistencia al cambio, aumentar la eficiencia y éxito en la organización, previendo las condiciones necesarias para generar el cambio y la disposición y compromiso de los miembros de la organización.

El deterioro del clima organizacional, la reducción de la productividad personal o corporativa y la ausencia de una identidad común, pueden ser las consecuencias más notorias del escaso compromiso de los trabajadores con la empresa, ello se complementa con algunas opiniones de expertos (Surdo, 1997):

- Los efectos del bajo sentido de identificación para con la organización son dos: Primero, la falta de motivación. El segundo es la disminución del profesionalismo del empleado.

- Una empresa donde la mayoría de personas tiene un bajo sentido de identificación nunca va a crecer, se va a quedar estancada y el ambiente interno sería terrible, pues se trabajaría con desánimo y desconfianza.

- Si no existe sentido de identificación, la gente y la compañía no pueden producir ni funcionar óptimamente, pues a la gente no le va a importar lo que suceda con la producción, no va a existir motivación en materia laboral y la gente va a trabajar únicamente por un salario.

\section{MATERIALES Y MÉTODOS}

El tipo de investigación es aplicada, cuyas características son descriptivas y correlaciónales. El diseño de la investigación es no experimental. La recolección de la información fue de corte transversal. La población de trabajadores que laboran en la Caja de Ahorro y Crédito del Cusco S.A. - Agencia Tacna es de 40 personas; distribuidos en el Área de Operaciones (12) y en el Área de Créditos (28). Se optó por desarrollar un censo, se encuestaron a las 40 personas. Los instrumentos fueron confeccionados por el investigador y su respectiva confiabilidad se dio a través del estadístico Alpha de Cronbach. Se aplicó la técnica de la encuesta y el instrumento del cuestionario. El procesamiento de los datos se hizo en base al software estadístico SPSS versión 20,0 en español. 


\section{RESULTADOS}

A través del software estadístico SPSS 20,0 se obtuvo el reporte de confiabilidad de los instrumentos aplicados; de donde:

Tabla $\mathrm{N}^{\circ} 1$

Alpha de Crombach: Resistencia al cambio

Estadísticos de fiabilidad
\begin{tabular}{|c|c|}
\hline $\begin{array}{c}\text { Alfa de } \\
\text { Cronbach }\end{array}$ & $\begin{array}{c}\mathrm{N} \text { de } \\
\text { elementos }\end{array}$ \\
\hline 0,892 & 25 \\
\hline
\end{tabular}
FUENTE: Reporte del SPSS 20,0

El valor obtenido de 0,892 refleja que la fiabilidad del instrumento "Resistencia al cambio" es adecuada.

\section{Tabla $\mathrm{N}^{\circ} 2$}

Alpha de Crombach: Identificación Institucional

Estadísticos de fiabilidad

\begin{tabular}{|c|c|}
\hline $\begin{array}{c}\text { Alfa de } \\
\text { Cronbach }\end{array}$ & $\begin{array}{c}\mathrm{N} \text { de } \\
\text { elementos }\end{array}$ \\
\hline 0,857 & 15 \\
\hline
\end{tabular}

El valor obtenido de 0,857 representa que la fiabilidad del instrumento "Identificación institucional" es adecuada.

\section{Tabla $\mathrm{N}^{\circ} 3$}

\section{Resistencia al cambio}

Estadísticos descriptivos

\begin{tabular}{|l|c|c|c|}
\hline Indicador & N & Media & Desv. típ. \\
\hline Resistencia al cambio & 40 & $\mathbf{1 0 3 , 6 7}$ & 9,082 \\
\hline
\end{tabular}

\begin{tabular}{|l|c|c|c|c|}
\hline \multirow{2}{*}{ Indicador } & \multicolumn{4}{|c|}{ Área } \\
\cline { 2 - 5 } & \multicolumn{2}{|c|}{ Área de Operaciones } & \multicolumn{2}{c|}{ Área de Créditos } \\
\cline { 2 - 5 } & \multicolumn{2}{|c|}{ Sexo } & \multicolumn{2}{c|}{ Sexo } \\
\cline { 2 - 5 } & Masculino & Femenino & Masculino & Femenino \\
\cline { 2 - 5 } & Media & Media & Media & Media \\
\hline Resistencia al cambio & 99 & $\mathbf{1 0 2}$ & $\mathbf{1 0 9}$ & 103 \\
\hline
\end{tabular}




\begin{tabular}{|c|c|c|c|c|c|}
\hline \multirow[t]{2}{*}{ Áreas } & & \multicolumn{3}{|c|}{ Resistencia al cambio } & \multirow[t]{2}{*}{ Tota } \\
\hline & & Regular & Baja & Muy baja & \\
\hline \multirow{3}{*}{ Área } & Área de Operaciones & 0 & 10 & 2 & 12 \\
\hline & Área de Créditos & 2 & 12 & 14 & 28 \\
\hline & Total & 2 & 22 & 16 & 40 \\
\hline
\end{tabular}

FUENTE: Cuestionario de "Resistencia al cambio"

La lectura de la tabla $\mathrm{N}^{\circ} 3$ permite observar que se obtuvo un valor promedio de 103,67 y una desviación típica de 9,082, que al ubicarlo en la escala de valoración ya definido, cae en la zona denominada "Resistencia al cambio baja"; por tanto, se concluye que el personal de la CMAC - Cusco S.A. - Agencia Tacna percibe que existiría un nivel bajo de resistencia ante posibles entornos de cambio en la organización; siendo el personal de sexo masculino el que presenta más bajos rasgos de resistencia. Al analizar por dimensión, son los "Factores asociados al saber/conocer" los que afectarían menos ante un entorno de posible cambio, siendo los "Factores organizacionales asociados al proyecto de cambio" los que afectarían más.

\section{Tabla $N^{\circ} 4$}

\section{Identificación institucional}

Estadísticos descriptivos

\begin{tabular}{|l|c|c|c|}
\hline Indicador & N & Media & Desv. típ. \\
\hline Identificación institucional & 40 & $\mathbf{6 2 , 5 5}$ & 7,056 \\
\hline
\end{tabular}

\begin{tabular}{|l|c|c|c|c|}
\hline \multirow{2}{*}{ Indicador } & \multicolumn{4}{c|}{ Área } \\
\cline { 2 - 5 } & \multicolumn{3}{|c|}{ Área de Operaciones } & \multicolumn{2}{c|}{ Área de Créditos } \\
\cline { 2 - 5 } & \multicolumn{2}{|c|}{ Sexo } & \multicolumn{2}{c|}{ Sexo } \\
\cline { 2 - 5 } & Masculino & Femenino & Masculino & Femenino \\
\cline { 2 - 5 } & Media & Media & Media & Media \\
\hline Identificación institucional & 55 & $\mathbf{6 6}$ & 62 & $\mathbf{6 5}$ \\
\hline
\end{tabular}

\begin{tabular}{|ll|c|c|c|c|}
\hline \multirow{2}{*}{ Áreas } & \multicolumn{3}{c|}{ Identificación institucional } & \multirow{2}{*}{ Total } \\
\cline { 3 - 5 } & Regular & Alta & Muy alta & \\
\hline \multirow{2}{*}{ Área } & Área de Operaciones & 4 & 2 & 6 & 12 \\
& Área de Créditos & 0 & 8 & 20 & 28 \\
\cline { 2 - 5 } & Total & 4 & 10 & $\mathbf{2 6}$ & 40 \\
\hline
\end{tabular}

FUENTE: Cuestionario "Identificación institucional" 
La lectura de la tabla $\mathrm{N}^{\circ} 4$ permite observar que se obtuvo un valor promedio de 62,55 y una desviación típica de 7,056 , que al ubicarlo en la escala de valoración ya definido, cae en la zona denominada "Identificación institucional muy alta"; por tanto, se concluye que el personal de la CMAC del Cusco S.A. - Agencia Tacna considera que se encuentra muy identificado con su entidad; siendo el personal de sexo femenino el que presenta mayores rasgos de ello. Al analizar por dimensión, es la "Realización" el aspecto más destacado, y siendo el "Compromiso" el aspecto a fortalecer.

\section{Prueba estadística de contraste}

Para la comprobación de influencia entre las variables, se aplicó el estadístico chi-cuadrado, cuyo reporte se presenta a continuación:

\section{a) Formulación de hipótesis}

$\mathrm{H}_{0}$ : No existe influencia entre la resistencia al cambio y la identificación del personal

$\mathrm{H}_{1}$ : Existe influencia entre la resistencia al cambio y la identificación del personal

b) Regla de decisión.

$p$ (p-value) $>0.05$ Se acepta la hipótesis nula $(\mathrm{Ho})$

$p(p$-value $)<0.05$ Se rechaza la hipótesis nula $(\mathrm{Ho})$

\section{c) Resultados del análisis estadístico}

\section{Pruebas de chi-cuadrado}

\begin{tabular}{|l|r|r|r|}
\hline Prueba Chi & \multicolumn{1}{|c|}{ Valor } & gl & \multicolumn{2}{|c|}{$\begin{array}{c}\text { Sig. asintótica } \\
\text { (bilateral) }\end{array}$} \\
\hline Chi-cuadrado de Pearson & $16,220^{a}$ & 4 &, 013 \\
Razón de verosimilitudes & 15,186 & 4 &, 005 \\
Asociación lineal por lineal & 1,811 & 1 &, 178 \\
N de casos válidos & 40 & & \\
\hline
\end{tabular}

a. 6 casillas $(66,7 \%)$ tienen una frecuencia esperada inferior a 5. La

frecuencia mínima esperada es ,20.

\section{d) Decisión}

Se obtuvo un valor de $p$ ( $p$-value $)=0,013$, que es menor que $a<0,05$ de significancia; por lo tanto se rechaza $\mathrm{H}_{0}$ y ello permite concluir que existe una influencia del nivel de resistencia al cambio sobre la identificación institucional del personal de la CMAC Cusco S.A. - Agencia Tacna. 


\section{CONCLUSIONES}

a) Se concluye que existe una influencia del nivel de resistencia al cambio sobre la identificación institucional del personal de la CMAC Cusco S.A. Agencia Tacna, con un nivel de confianza del 95\%.

b) Se concluye que el personal de la CMAC del Cusco S.A. - Agencia Tacna percibe que existe un nivel bajo de resistencia ante posibles entornos de cambio en la organización; siendo los "Factores asociados al saber/conocer" los que afectan menos y los "Factores organizacionales asociados al proyecto de cambio" los que afectan más.

c) Se concluye que el personal de la CMAC del Cusco S.A. - Agencia Tacna considera que se encuentra muy identificado con su entidad; siendo la "Realización" la dimensión más destacada, y el "Compromiso" el aspecto a fortalecer.

\section{REFERENCIAS BIBLIOGRÁFICAS}

- Alles, M. (2000). Dirección estratégica de recursos humanos. Editorial Granica. Buenos Aires (Argentina).

- Alemán, D. y Böhrt, R. (2004). Análisis de la resistencia al cambio en un supermercado.

http://www.ucb.edu.bo/publicaciones/ajayu/v2n2/v2n2a2.pdf

URL

- Andreu, R., Ricart, J. y Valor, J. (1995). La organización en la era de la información: Aprendizaje, innovación y cambio. Editorial Estudios y Ediciones IESE. México. II Edición.

- Biasca, R. (2005). Gestión de cambio: el modelo Básico- Organizational Improvement And Change. URL. https://books.google.com. pe/books?id=TAhNf5fV3KAC\&printsec=frontcover \&hl=es\&source=gbs_ge_summary_r\&cad $=0 \# v=$ onepage \&q\&f=false

- Benavides, O. (2002); Competencias y competitividad: Diseño para organizaciones latinoamericanas. Editorial McGraw-Hill. Colombia. Pág. 145.

- Blake, R., Mouton, J. y MC canse, A. (1997); La estrategia para el cambio organizacional. Editorial Addison Wesley.

- Cabrera, A. y Onache, J. (2002). Dirección estratégica de personas. Editorial Prentice Hall. España: Financial Times.

- Chiavenato, I. (2000). Administración de recursos humanos. Editorial Internacional Thomson, S.A. Colombia.

- Contreras, A. y Hernández E. (2011). Antecedentes teóricos y niveles de análisis de la identidad organizacional. Consultado el 28 de agosto 2013, URL http://www.redalyc.org/pdf/2033/203318388009.pdf

- Davenport, T. (2006). Capital humano. Creando ventajas competitivas a través de las personas. Ediciones DEUSTO. España.

- Davis, K., y Newstrom, J. (1999). Comportamiento humano en el trabajo. Editorial McGraw-Hill. México.

- Dorta, A. (2011). Resistencia al Cambio. URL http://centrodedesarrollogerencial.blogspot.com/2011/01/resistencia-alcambio.html

- Drucker, P. (2002). La gerencia. Editorial El Ateneo. Buenos Aires (Argentina). 
- Echezarreta, C. (2007). La Concurrencia de Intereses del Trabajador y la Empresa. URL https://estudioechezarreta.wordpress.com/2007/07/23/laconcurrencia-de-intereses-del-trabajador-y-la-empresa/

- García, V. y Macias, H. (2001). Cambio Organizacional en las Empresas Tabasqueñas.

URL http://www.publicaciones.ujat.mx/publicaciones/hitos/ediciones/17/cambioorg .pdf.

- Goncalves, A. (2000). Fundamentos del clima organizacional. Sociedad Latinoamericana para la calidad (SLC).

- Gordon, J. (1997). Comportamiento organizacional. Editorial Prentice Hall Hispanoamericana. México. V Edición.

- Gross, M. (2010). Cuatro Modelos de Comportamiento Organizacional. URL http://manuelgross. bligoo.com/content/view/780384/Cuatro-Modelos-deComportamiento-Organizacional.html

- Hellriegel, D. (2004). Administración. Editorial International Thomson. México. XI Edición. URL

- Ibáñez, M. (2002). Comportamiento organizacional. Universidad Ricardo Palma.

- Ivancevich, J., Konopaske, R. y Matteson, M. (2005). Comportamiento organizacional". Editorial McGraw-Hill. México.

- Koontz, H. Hld y Werkrich. (1999). Administración: Una perspectiva global. Editorial McGraw-Hill. México. Il edición.

- Loli, A. (2007). Compromiso organizacional de los trabajadores de una Universidad Pública.

URL http://sisbib.unmsm.edu.pe/bibvirtualdata/publicaciones/indata/vol10_n2/a06. pdf

- Luperdi, C. (2007). Resistencia AL CAMBIO por Carlos. URL http://manuelgross.bligoo.com/content/view/620641/Tres-causas-de-laresistencia-al-cambio-organizacional.html

- Quintero, T. (2003). Comportamiento Organizacional. URL http://www.gestiopolis.com/recursos/documentos/fulldocs/ger1/cotahis.htm

- Reyes, A. (s/f). Cambio Organizacional. URL http://www.monografias.com/trabajos13/cborgdef/cborgdef.shtml

- Robbins S. (2004). Comportamiento organizacional. Editorial Prentice Hall. México. X Edición.

Recibido: $22 / 08 / 2015$

Aceptado para publicación:

$17 / 11 / 2015$ 